\title{
Untersuchungsanlage und Auswertungsmethodik
}

\subsection{Untersuchungsanlage}

Bevor im Folgenden die Untersuchungsanlage erläutert wird, ein Hinweis zur Repräsentativität der Befunde. Die Ergebnisse qualitativer Studien sind nicht im statistischen, wohl aber im psychologischen Sinne repräsentativ. Durch die Flexibilität des qualitativ-ethnologischen Forschungsansatzes mit non-direktiven Methoden wird eine hohe Inhaltsvalidität und Unverfälschtheit der Ergebnisse erreicht, ohne allerdings statistisch repräsentative Aussagen über Prozentverteilungen machen zu können. Ziel qualitativer Forschung ist es vielmehr, alle psychologisch wirksamen Einflussfaktoren bei einem Thema (z. B. Einstellungen, Erwartungen, Emotionen, Motive) offenzulegen und verstehend zu beschreiben. Im Unterschied zu quantifizierenden Methoden ist dafür bereits eine relativ kleine Stichprobe ausreichend.

Die vorliegende Studie basiert auf 80 qualitativen Einzelinterviews von Geflüchteten im Alter von 14 bis 17 Jahren, die seit 2015 nach Deutschland gekommen sind. Sie ist Teil eines größeren Forschungsprogramms zu jugendlichen Geflüchteten, bei dem das SINUS-Institut neben der Deutschen Kinder- und Jugendstiftung (DKJS) auch mit der Bundeszentrale für gesundheitliche Aufklärung (BZgA) kooperierte. Dabei gab es gemeinsame wie auch institutionenspezifische Erkenntnisinteressen: Die Projektpartner förderten ein gemeinsames Modul zur Lebenswelt jugendlicher Geflüchteter, in dem Fragen dazu gestellt wurden, wie die Jugendlichen den Alltag in Deutschland (er)leben (ca. 25 Minuten der Befragungszeit). Darüber hinaus erhob das SINUS-Institut für die BZgA Daten zum Thema Partnerschaft, Familienplanung und Sexualität (ca. 45 Minuten der Befragungszeit) und für die DKJS Daten zur Berufsorientierung jugendlicher Ge- 
flüchteter (ca. 20 Minuten der Befragungszeit). Die Datenerhebung fand zwischen August 2017 und Februar 2018 statt.

Die Jugendlichen hatten ca. zwei Wochen vor Durchführung des Interviews einen nicht-standardisierten Selbstausfüller-Fragebogen zum Thema „So bin ich, das mag ich“ erhalten. Diese „Hausaufgabe“ erfüllte neben dem Gewinn von inhaltlichen Erkenntnissen auch den Zweck, Barrieren und womöglich Skepsis oder gar Ängste der Jugendlichen (und ihrer Eltern) im Vorfeld des Gesprächstermins abzubauen. Es wurden auch einige Fragen gestellt, die im weiteren Sinne für die Berufsorientierung relevant sind:

- Was ist dein Traumberuf? Was ist das Tolle an diesem Beruf?

- Wo bist du im Internet unterwegs (z. B. Blogs, etc.)?

- Was sind für dich die wichtigsten Dinge der Welt?

- Wie möchtest du später leben? Was machst du dann?

- Wie sieht für dich die ideale Partnerschaft aus?

- Wofür interessierst du dich?

- Was gibt deinem Leben Sinn?

Die Jugendlichen wurden zudem gebeten, für sie bedeutsame Eindrücke/Bilder aus ihrem Alltag fotografisch festzuhalten. Hierfür wurden ihnen analoge Einwegkameras zur Verfügung gestellt. Die Jugendlichen erhielten frankierte Umschläge für die Rücksendung an SINUS.

\section{Die Gespräche wurden von Interviewerinnen und Interviewern aus dem gleichen Herkunftsland wie die Interviewten durchgeführt - in der Muttersprache der Jugendlichen.}

Das Forschungskonzept setzte besondere Qualifikationen der Interviewenden voraus. Sie mussten eine fachliche Nähe zum Untersuchungsthema haben und mit Grundlagen der Interviewtechnik vertraut sein. Alle eingesetzten Interviewerinnen und Interviewer wurden im Rahmen eines ganztätigen Praxis-Workshops vertiefend methodisch geschult und inhaltlich an das Projekt herangeführt. Die Interviews wurden jeweils von gleichgeschlechtlichen Interviewenden in der Muttersprache der Jugendlichen geführt. Mit einigen dieser Interviewenden hat das SINUS-Institut bereits im Zuge vorheriger Studien zusammengearbeitet.

Die Gespräche selbst wurden, um jeden Informationsverlust zu vermeiden, auf Tonband aufgezeichnet. Die Audioaufzeichnungen wurden dann durch die Interviewerinnen und Interviewer ins Deutsche übersetzt und verschriftlicht. Teil dieser Protokolle waren auch Hinweise auf kulturelle und sprachliche Spezifika (z. B. Übersetzung von kulturspezifischen Redewendungen). 


\section{Zentrale Merkmale bei der Stichprobenziehung waren Herkunftsland und Geschlecht.}

Unter Berücksichtigung der offiziellen Statistik des Bundesamts für Migration und Flüchtlinge (BAMF) zu minderjährigen Geflüchteten im Alter von 14 bis 17 Jahren war ursprünglich für die Studie folgender Stichprobenansatz entwickelt worden:

\begin{tabular}{|c|c|c|c|c|c|c|c|}
\hline \multicolumn{2}{|c|}{$\begin{array}{c}\text { Syrien } \\
\mathrm{N}=24\end{array}$} & \multicolumn{2}{c|}{$\begin{array}{c}\text { Afghanistan } \\
\mathrm{N}=24\end{array}$} & \multicolumn{2}{c|}{$\begin{array}{c}\text { Irak } \\
\mathrm{N}=20\end{array}$} & \multicolumn{2}{c|}{$\begin{array}{c}\text { Eritrea } \\
\mathrm{N}=12\end{array}$} \\
\hline Männer & Frauen & Männer & Frauen & Männer & Frauen & Männer & Frauen \\
\hline 16 & 8 & 16 & 8 & 12 & 8 & 8 & 4 \\
\hline
\end{tabular}

Hierfür stellte das SINUS-Institut eine Anfrage beim Referat Statistik des BAMF zu den zehn zugangsstärksten Herkunftsländern der 14- bis 17-jährigen Erstantragsteller (Zeitraum: 1.1.2016 bis 30.09.2016). Aufgrund der weiter unten ausgeführten Herausforderungen bei der Feldarbeit weicht die tatsächliche Stichprobe vom geplanten Zuschnitt wie folgt ab:

\begin{tabular}{|c|c|c|c|c|c|c|c|}
\hline \multicolumn{2}{|c|}{$\begin{array}{c}\text { Syrien } \\
\mathrm{N}=31\end{array}$} & \multicolumn{2}{c|}{$\begin{array}{c}\text { Afghanistan } \\
\mathrm{N}=26\end{array}$} & \multicolumn{2}{c|}{$\begin{array}{c}\text { Irak } \\
\mathrm{N}=20\end{array}$} & \multicolumn{2}{c|}{$\begin{array}{c}\text { Eritrea } \\
\mathrm{N}=3\end{array}$} \\
\hline Männer & Frauen & Männer & Frauen & Männer & Frauen & Männer & Frauen \\
\hline 19 & 12 & 18 & 8 & 12 & 8 & 3 & - \\
\hline
\end{tabular}

Von einer Berücksichtigung des Merkmals „Bildung“ wurde bei der Stichprobenbildung abgesehen, da eine verlässliche Erfassung und Kategorisierung des tatsächlichen Bildungsstands kaum möglich ist: Zum einen fehlt eine herkunftslandübergreifende Vergleichbarkeit der Schulformen, zum anderen haben die Jugendlichen aufgrund der Situation im Herkunftsland und/oder wegen ihrer Flucht in der Vergangenheit nicht durchgängig eine Schule besuchen können. Auch der Besuch einer bestimmten Bildungseinrichtung hier in Deutschland spiegelt nur bedingt den Bildungsstand der Teilnehmenden, da Sprachkenntnisse und Dauer des Aufenthalts in Deutschland eine ebenso wichtige Rolle spielen.

Die von den befragten Jugendlichen derzeit besuchten Bildungseinrichtungen umfassen alle einschlägigen Schultypen von Haupt-, Real- und Gesamtschule bis Gymnasium und Berufsschule.

18 Befragte erklärten, spezifische Sprachförderungsprogramme zu besuchen. Die genauen Bezeichnungen hierfür unterscheiden sich von Bundesland zu Bundesland. In Baden-Württemberg wird beispielsweise von „Vorbereitungsklassen“ und „Vorbereitungskursen“ gesprochen und in Berlin von „Lerngruppen für Neuzugänge ohne Deutschkenntnisse“. Solche Fördermaßnahmen sind auch 
nicht einheitlich organisiert: „Insgesamt handelt es sich nicht um klar abgrenzbare Formen, sondern um ein Kontinuum an Organisationsformen mit unterschiedlich abgestuften Anteilen von Sprachförderung und Unterricht in einer Regelschulklasse [...]“ (Massumi et al. 2015, 44). In welcher Organisationsform von Vorbereitungsklassen sich die befragten Jugendlichen zum Zeitpunkt der Interviews befanden, wurde im Rahmen dieser Studie nicht erfasst. Im Folgenden wird verallgemeinernd von „Vorbereitungsklassen“ gesprochen.

\section{Die Interviews wurden in verschiedenen Regionen Deutschlands geführt und kamen über eine Vielzahl an Zugängen zustande.}

Es wurden sowohl unbegleitete Jugendliche interviewt als auch Mädchen und Jungen, die mit ihren Eltern oder anderen volljährigen Familienmitgliedern nach Deutschland gekommen sind. Das Spektrum der Wohnformen war vielfältig: Darunter befanden sich Erstaufnahmeeinrichtungen, Anschlussunterbringungen, betreute Wohnformen, Kinder- und Jugendheime, private Wohnungen mit den Eltern oder anderen Verwandten, private Wohnungen für sich alleine und Wohnungen in Pflegefamilien.

Die Interviews wurden in folgenden Orten durchgeführt:

- Berlin

- Bremen

- Baden-Württemberg: Auenwald, Baden-Baden, Backnang, Durmersheim, Eberbach, Gernsbach, Heidelberg, Ötigheim, Ottersweier, Stuttgart und Weinheim

- Brandenburg: Stadt Brandenburg

- Nordrhein-Westfalen: Bocholt, Bochum, Datteln, Dortmund, Essen, Herne und Mülheim

- Hessen: Haiger

- Sachsen-Anhalt: Hettstedt, Oschersleben und Weißenfels

- Schleswig-Holstein: Kiel

Die Rekrutierung der Jugendlichen erfolgte über folgende Wege:

- Professionelle Felddienstleister

- Netzwerk der SINUS-Akademie: hauptsächlich Akteure der offenen und verbandlich organisierten Jugendhilfe

- Netzwerk der Deutschen Kinder- und Jugendstiftung (Mitarbeiterinnen und Mitarbeiter der DKJS oder der regionalen Servicebüros der DKJS, ehren- wie auch hauptamtliche Mitarbeiterinnen und Mitarbeiter in Projekten aus dem 
Programm der DKJS, Mitarbeiterinnen und Mitarbeiter in Ämtern bzw. Behörden)

- Netzwerk des SINUS-Instituts (freie Rekrutierungs- und Netzwerkkräfte)

- Interviewerinnen und Interviewer der Studie

\section{Die Durchführung der Interviews war aus mehreren Gründen schwierig.}

Im Verlauf der Feldphase ergaben sich verschiedene Herausforderungen:

- So waren beispielsweise Jugendliche nach der Zusage zum Interview unter ihrer Mobilnummer nicht mehr erreichbar (Nummer ungültig).

- Andere befanden sich zunächst in einer Gemeinschaftsunterkunft, haben diese aber während der Rekrutierungsphase verlassen. Der neue Aufenthaltsort konnte (u. a. aus Datenschutzgründen) nicht ermittelt werden.

- Einige Jugendliche wurden zwischenzeitlich abgeschoben, wiederum andere sind in ein anderes Land umgezogen.

- Auch waren oftmals die Kontaktpersonen in den Unterkünften oder Jugendeinrichtungen, die ursprünglich die Teilnahme zugesagt hatten, aus unterschiedlichen Gründen nicht (mehr) vor Ort. Falls Vertretungen nicht informiert wurden und/oder Vorbehalte hatten, kamen Interviews ebenfalls nicht zustande.

- Bei zahlreichen Probanden haben die Eltern der Jugendlichen die Teilnahme untersagt. In den meisten Fällen bestand nach Auskunft der Rekrutiererinnen und Rekrutierer Misstrauen gegenüber Anonymitätszusicherungen sowie Angst vor möglichen Konsequenzen im Asylverfahren.

- Erschwerend bei der Durchführung kam hinzu, dass trotz vorheriger Bitte nicht alle Interviews unter vier Augen geführt werden konnten, weil ohne Anwesenheit von Familienmitgliedern sonst die Teilnahme abgesagt worden wäre. Die Interviewenden haben in den Interviewtranskripten vermerkt, an welchen Stellen die Anwesenheit Dritter ihrer Einschätzung nach Einfluss auf das Antwortverhalten hatte.

- Die ohnehin bereits zeitaufwändige Kontaktaufnahme verzögerte sich auch dadurch, dass zwischen Erstkontakt und endgültiger Absage oft bis zu sechs Wochen lagen und die Kommunikation über mehrere zwischengeschaltete Instanzen laufen musste. Die nötigen Nachrekrutierungen benötigten dann wieder erneuten zeitlichen Vorlauf.

- Die Kontaktaufnahme zu Jugendlichen aus Eritrea, vor allem zu Mädchen, gestaltete sich äußerst aufwändig und kompliziert. Bei den Mädchen kam es trotz Erstkontakten zu keiner Terminvereinbarung. Im Verlauf der Feldphase wurde daher aus forschungsökonomischen Gründen beschlossen, diese Gruppe nicht zu befragen. 


\subsection{Auswertungsmethodik}

Die Daten wurden mit der sozialwissenschaftlichen Methode der qualitativen Inhaltsanalyse ausgewertet. Die Arbeitsschritte hierfür lassen sich wie folgt skizzieren: Die Interviews wurden zunächst ins Deutsche übersetzt und transkribiert. Im Anschluss wurden die Transkripte vom Forscher-Team des SINUS-Instituts mithilfe eines Computerprogramms zur Analyse qualitativer Daten (QDA Miner) codiert und ausgewertet. Codieren bedeutet, im Zuge der Datensichtung alle relevanten Textstellen passenden Kategorien zuzuordnen. Mit der Codierung der Transkripte wird das Ziel verfolgt, die Komplexität des Datenmaterials zu reduzieren, indem mithilfe eines Kategoriensystems diejenigen Aspekte markiert werden, die für die Auswertung bedeutsam sind. Dabei werden die Transkripte mit Struktur-Codes versehen, die den zentralen Themenbereichen des Interviewleitfadens bzw. der Untersuchung entsprechen - z. B. Freizeitinteressen, Werte, Beziehungen, Freundeskreis, Zukunftsperspektive, Berufswunsch, Kenntnisse über das Ausbildungssystem, Informationsbedarfe bei der beruflichen Orientierung etc. Daran anschließend werden die Transkripte im Zusammenhang analysiert und zentrale Textstellen markiert (In-Vivo-Codierung) sowie inhaltlich interpretiert (Zuweisung eines interpretativen Codes). Da in ein und derselben Textstelle oft unterschiedliche Themen angesprochen werden, ist es möglich und sinnvoll, diese mit verschiedenen Codes zu versehen. Ein Beispiel:

\begin{tabular}{|c|c|c|}
\hline Struktur-Code & In-Vivo-Code & Interpretativer Code \\
\hline Beziehungen & $\begin{array}{l}\text { Eine Partnerin vor der Ehe ist } \\
\text { in meiner Religion nicht } \\
\text { erlaubt. Meine Ehefrau sollte } \\
\text { auf jeden Fall Muslimin sein. }\end{array}$ & $\begin{array}{l}\text { - } \text { Multikulturalismus negativ } \\
\text { - } \quad \text { Außereheliche Beziehung negativ } \\
\text { - }\end{array}$ \\
\hline
\end{tabular}

Die meisten interpretativen Codes der vorliegenden Studie wurden aus den Befunden selbst hergeleitet. Die einzigen Codes, die nicht im Zuge der Auswertung abgeleitet wurden, sind die verwendeten Berufskategorien. Hier wurde auf eine Berufsklassifikation der Bundesagentur für Arbeit zurückgegriffen (Bundesagentur für Arbeit 2011).

Aus dem ersten Codierdurchlauf aller Interviews resultierten unzählige In-VivoCodes sowie ca. 150 interpretative Codes. Um sicherzustellen, dass die Codes plausibel sind, wurde das Datenmaterial von mehreren Forscherinnen und Forschern unabhängig voneinander codiert. In der weiteren Auswertung wurden nur diejenigen Codes verwendet, bei denen die codierenden Personen weitgehend übereinstimmten (hier spricht man vom Gütekriterium der „Intercoder-Reliabilität"). In einem zweiten Codierlauf wurden dann redundante Codes zusammen- 
gefasst. Insgesamt ergaben sich so ca. 100 finale Codes. Einige Beispiele für diese Codes:

- Bildung und Beruf (z. B. Anerkennung, Bedeutung der Familie bei Berufswunsch, Weiterbildung etc.)

- Migration und Integration (z. B. Aufenthaltsstatus, deutsche Sprache, Diskriminierung etc.)

- Gesundheit, Familienplanung und Sexualität (z. B. Beziehungen im Umfeld, Geschlechterrollen: traditionelle/moderne, LGBTQ etc.)

- Werte (z. B. Ansehen, Aufgeschlossenheit, Authentizität etc.)

Die computergestützte Codierung bzw. Analyse ermöglicht es, nach Zusammenhängen in den Daten zu suchen. Um eine sinnvolle Gruppierung der Befunde zu ermöglichen, wurden für jeden Fall in QDA Miner folgende demografische Merkmale hinterlegt:

1. Geschlecht

2. Alter

3. Religion

4. Herkunftsland

5. Bisherige Aufenthaltsdauer in Deutschland

6. Besuchte Schulform in Deutschland

7. Besuchte Schulform im Herkunftsland

8. Bundesland

9. Haushalt und Art der Unterbringung

Im Rahmen der Datenauswertung konnten auf diese Weise viele allgemeine Befunde ermittelt werden, die auf die Mehrheit der Befragten zutreffen (weitgehend unabhängig von demografischen oder soziokulturellen Merkmalen). Ein Beispiel:

- Unabhängig von demografischen Merkmalen schreiben alle befragten Jugendlichen der eigenen beruflichen Zukunft eine große Bedeutung zu.

Es zeigte sich aber auch, dass viele Einstellungen und Sichtweisen der jungen Geflüchteten mit demografischen Merkmalen oder bestimmten Rahmenbedingungen (der Meso- und Mikro-Ebene) zusammenhängen. Beispiele:

- Die befragten jungen Frauen interessieren sich stärker als die jungen Männer für Berufe, die einen Studienabschluss voraussetzen. 
- Unsichere Bleibeaussichten wurden vor allem von den jungen afghanischen Geflüchteten in den Interviews thematisiert.

Andere (interessante) Befunde sind fallspezifisch, d. h. können weder auf die Gesamtheit noch auf bestimmte Untergruppen der Stichprobe verallgemeinert werden.

\subsection{SINUS-Mindsets von jugendlichen Geflüchteten im Alter von 14 bis 17 Jahren}

Neben Befunden zur beruflichen Orientierung umfasst der vorliegende Bericht auch eine Werteanalyse der Befragten. Bilsky und Schwartz definieren „Werte“ entlang fünf formaler Kriterien (1987, 551):

1. Werte sind Konzepte und Überzeugungen

2. bezüglich wünschenswerter Zustände oder Verhaltensweisen,

3. die über eine spezifische Situation hinausgehen,

4. die die Auswahl oder die Bewertung von Verhalten und Ereignissen steuern, und

5. die nach relativer Bedeutung geordnet werden.

Die Mehrheit der befragten Jugendlichen unterscheidet zwischen wünschenswerten und nicht wünschenswerten Zuständen und Verhaltensweisen. Sie glauben, dass bestimmte Sachverhalte, Konzepte, Überzeugungen und Beziehungsqualitäten über die individuellen Umstände hinausreichen und Grundlage für ,das gute Leben" an sich darstellen. Fragt man die Jugendlichen, was ihnen im Leben am wichtigsten ist, werden am häufigsten Familie, Vertrauen, Freundschaft, Bildung, physische und soziale Sicherheit sowie Religion genannt. Weitere häufig erwähnte oder implizierte Werte sind Nächstenliebe, Menschlichkeit, Verantwortung, Heimat, Toleranz, Respekt, Unabhängigkeit und Erfolg. Sieht man von den zwei letztgenannten ab, so sind alle diese Werte pro-sozial und/oder stabilitätsorientiert.

Zusätzlich zu diesem allgemein geteilten Werte-Set äußern die Jugendlichen explizit wie implizit eine Bandbreite differenzierender Vorstellungen, Ziele und Orientierungen. Hierunter fallen auch Werte, die primär individuellen und nicht kollektiven Interessen dienen, wie Karriere, Prestige, Geld und Luxus. Zudem werden Werte genannt, die eher Risikoaffinität als Sicherheitsorientierung spiegeln, wie beispielsweise Freizügigkeit und Hedonismus. Und nicht zuletzt werden auch sich widersprechende Vorstellungen angeführt, die sich z. B. auf sozio-poli- 
tische Positionen wie Universalismus versus Patriarchat oder auf gegensätzliche Umgangsarten mit kulturellen Unterschieden wie Bi- und Multikulturalismus versus ethnisch-kultureller Abgrenzung beziehen. Eine detaillierte Beschreibung des Werteuniversums jugendlicher Geflüchteter war Gegenstand des Untersuchungsteils für die Bundeszentrale für gesundheitliche Aufklärung (Publikation voraussichtlich im Frühjahr 2019).

Wenn man die strukturellen Zusammenhänge zwischen diesen Werten untersucht, zeigen sich bestimmte Werte-Cluster, und es schält sich eine Typologie unterschiedlicher Lebensorientierungen heraus. Die auf diese Weise identifizierten Typen sind nicht identisch mit sozialen Milieus - dazu fehlt ihnen die lebensweltliche Tiefenschärfe, denn die Jugendlichen, die sie beschreiben, sind noch zu kurz in Deutschland, um sich im sozialen Raum etabliert zu haben. Dementsprechend konnten viele Fragen nicht gestellt werden, die zentral sind, um ein soziales Milieu zu bestimmen (z. B. soziale und Partnerbeziehungen, Alltagsästhetik, Konsumpräferenzen, Meinungen und Einstellungen zur deutschen Gesellschaft und Kultur, etc.). Anhand der erhobenen Daten ist es aber möglich, dominante soziale Orientierungen bzw. Mindsets zu unterscheiden.

Diese Mindsets kann man in einem zweidimensionalen Modell darstellen, bei dem die X-Achse die normative Grundorientierung und die Y-Achse die sozialen Aspirationen, d. h. die Projektion der angestrebten Positionierung im gesellschaftlichen Gefüge, abbildet (Abb. 1).

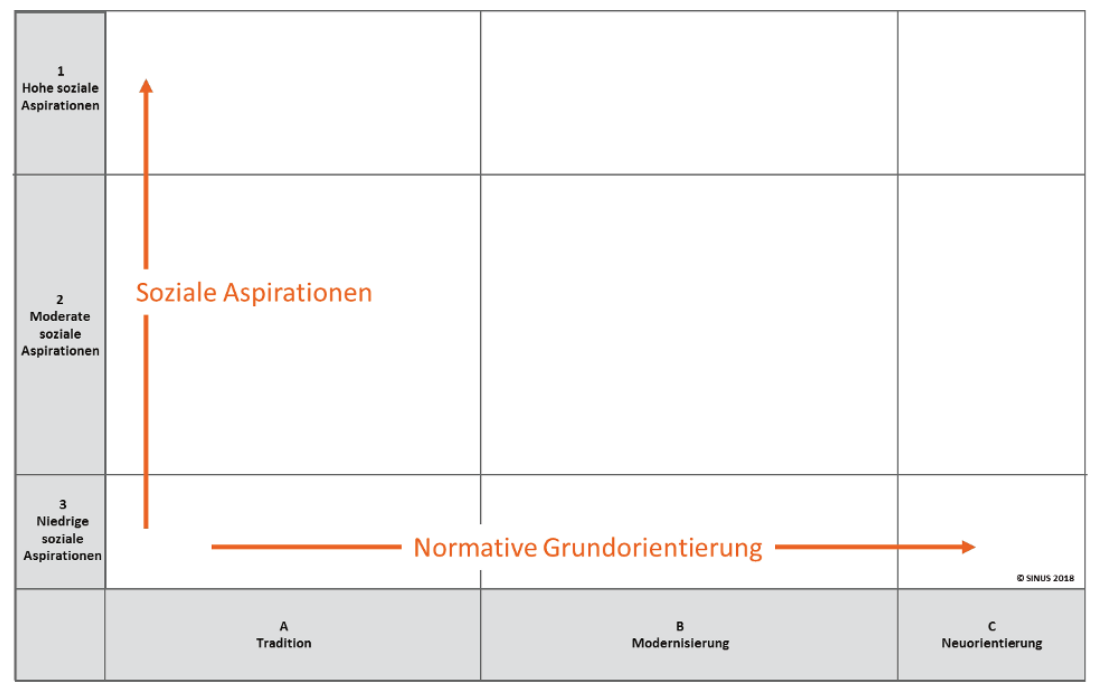

Abb. 1 Mindset-Dimensionen 
Die X-Achse des obenstehenden Modells skaliert die Wertevorstellungen und sozialen Normen. In Anlehnung an die Migranten-Milieustudien von SINUS aus den Jahren 2008 und 2017 ist diese Achse in drei Abschnitte unterteilt: „Tradition““ „Modernisierung,“ und „Neuorientierung“ (vgl. Flaig und Schleer 2018, 119). Auf der linken Seite befindet sich der Bereich „Tradition“, gekennzeichnet durch eine starke Verbundenheit mit überkommenem Wissen und kultureller Tradition als Wert an sich, durch den Wunsch, die Werte der Herkunftsgesellschaft zu erhalten, und auch durch eine virulente Unsicherheit, wie man sich in der Mehrheitsgesellschaft verhalten und neu positionieren soll. Im breiten Mittelfeld der Skala liegt der Bereich „Modernisierung“. Hier dominiert der pragmatische Wunsch, die Traditionen und Normen des Herkunftslandes an die des Aufnahmelandes anzupassen und auch neue Orientierungen zu übernehmen, mit dem Ziel, Akzeptanz und sozioökonomischen Aufstieg in der Mehrheitsgesellschaft zu erreichen. Am rechten Ende der Skala findet sich der Bereich „Neuorientierung“. Hier steht der Wunsch im Vordergrund, die Bedeutung und Geltung von Werten und Normen sowohl der Herkunfts- als auch der Aufnahmegesellschaft für sich selbst auszutesten. Jugendliche mit dieser Grundorientierung haben eine starke Affinität zu neuen und alternativen Lebensstilen. Basierend auf dem in der Studie „Wie ticken Jugendliche?" (Calmbach et al. 2016) entwickelten Modell ist es gelungen, das Wertespektrum auf der X-Achse des vorliegenden Modells einzuordnen.

Die Y-Achse bildet die Ziele und Erwartungen hinsichtlich der gesellschaftlichen Stellung ab. Das obere Ende wird durch das Streben nach tertiärer Bildung und Höherqualifizierung, nach hochrangigen und prestigeträchtigen Berufen definiert - oft in Kombination mit einem ausgeprägten Sinn für Selbstwirksamkeit und optimistischen Zukunftserwartungen. Im mittleren Bereich stellt das Erreichen einer „Normalbiografie“ das Ziel dar, z. B. durch den erfolgreichen Abschluss einer Ausbildung und das Ergattern eines ,anständigen“ (häufig handwerklichen) Berufs. Dabei sind die Jugendlichen bereit, für einen (moderaten) beruflichen Aufstieg und für finanzielle Sicherheit hart zu arbeiten. Am unteren Ende der Skala findet man eher geringe Bildungs- und Karriereambitionen, manchmal verbunden mit einer positiv-akzeptierenden Haltung gegenüber den Gegebenheiten, manchmal auch eher resignativ.

Als Ergebnis der inhaltlichen Analyse zeigen sich vier verschiedene wertebasierte Mindsets:

I. Moving Up: traditionsbezogen und sicherheitsbedacht, mit starker Aufstiegsorientierung

II. Breaking Free: veränderungsbereit, experimentalistisch und selbstverwirklichungsorientiert 
III. Holding On: traditionsverhaftet und in-group-orientiert, oft verunsichert und pessimistisch

IV. Blending In: stabilitätsorientiert, anpassungswillig, mit moderaten sozialen Zielen

Für jedes Mindset konnten fünf bis sechs Jugendliche identifiziert werden, die seine Charakteristik beispielhaft vertraten. Die Mehrzahl der Jugendlichen befand sich aber in Übergangsbereichen, weswegen die Mindsets auch als heuristische Idealtypen $\mathrm{zu}$ verstehen sind. Um diesem Faktum Rechnung zu tragen, sind bei der Visualisierung der Mindsets im Koordinatenkreuz große Überlappungsflächen eingezeichnet (Abb. 2).

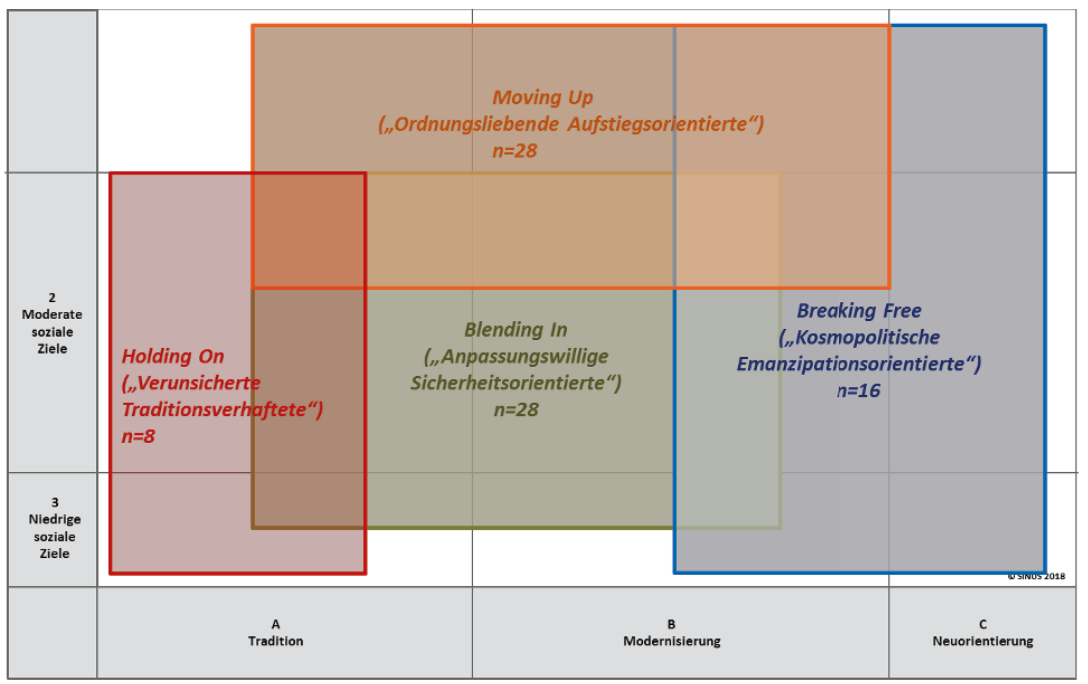

Abb. 2 Mindset-Typologie

In den folgenden Kapiteln wird die Berufsorientierung jugendlicher Geflüchteter auch differenziert nach diesen Mindsets betrachtet. 


\subsubsection{Mindset-Profil: Moving Up}
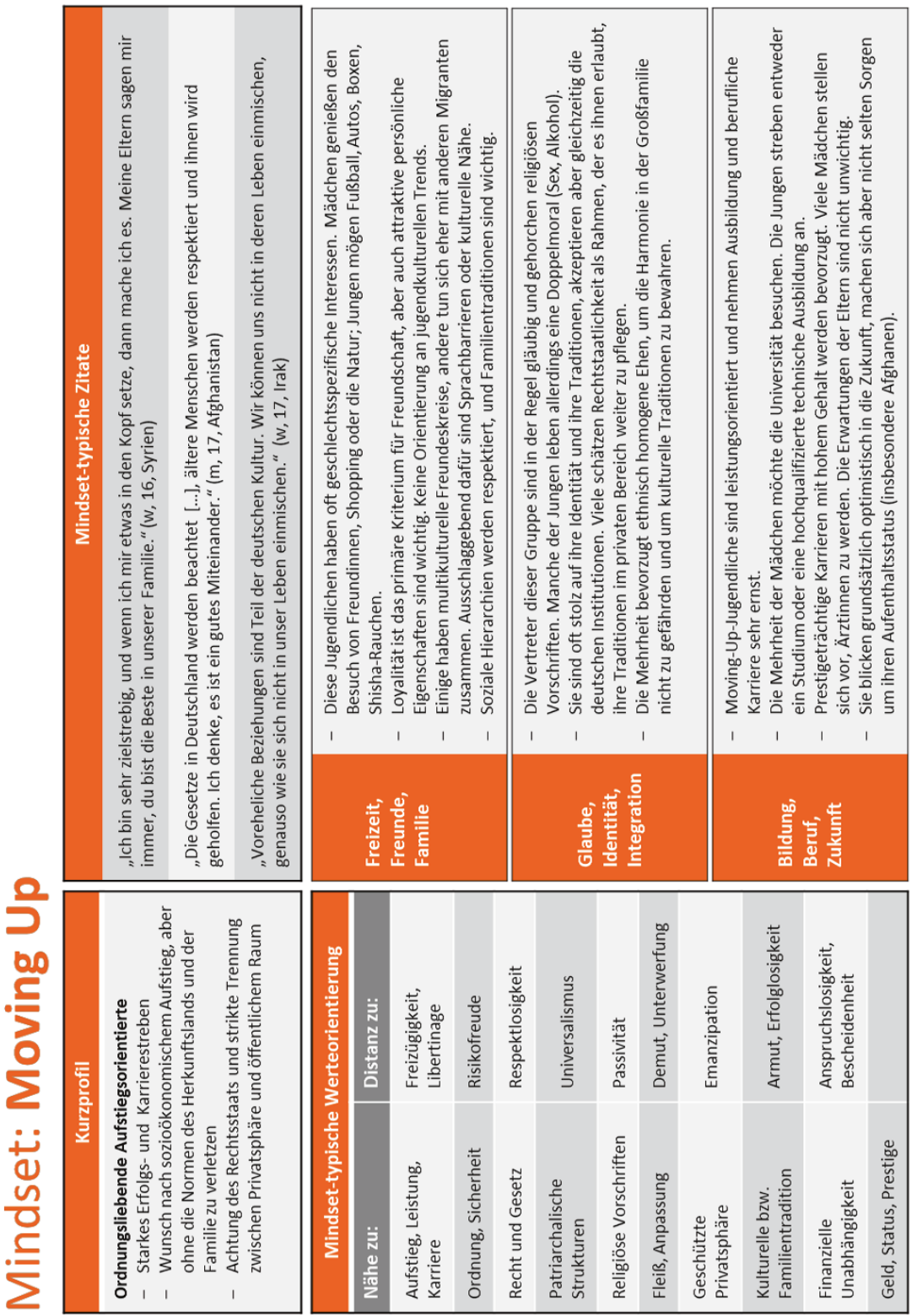

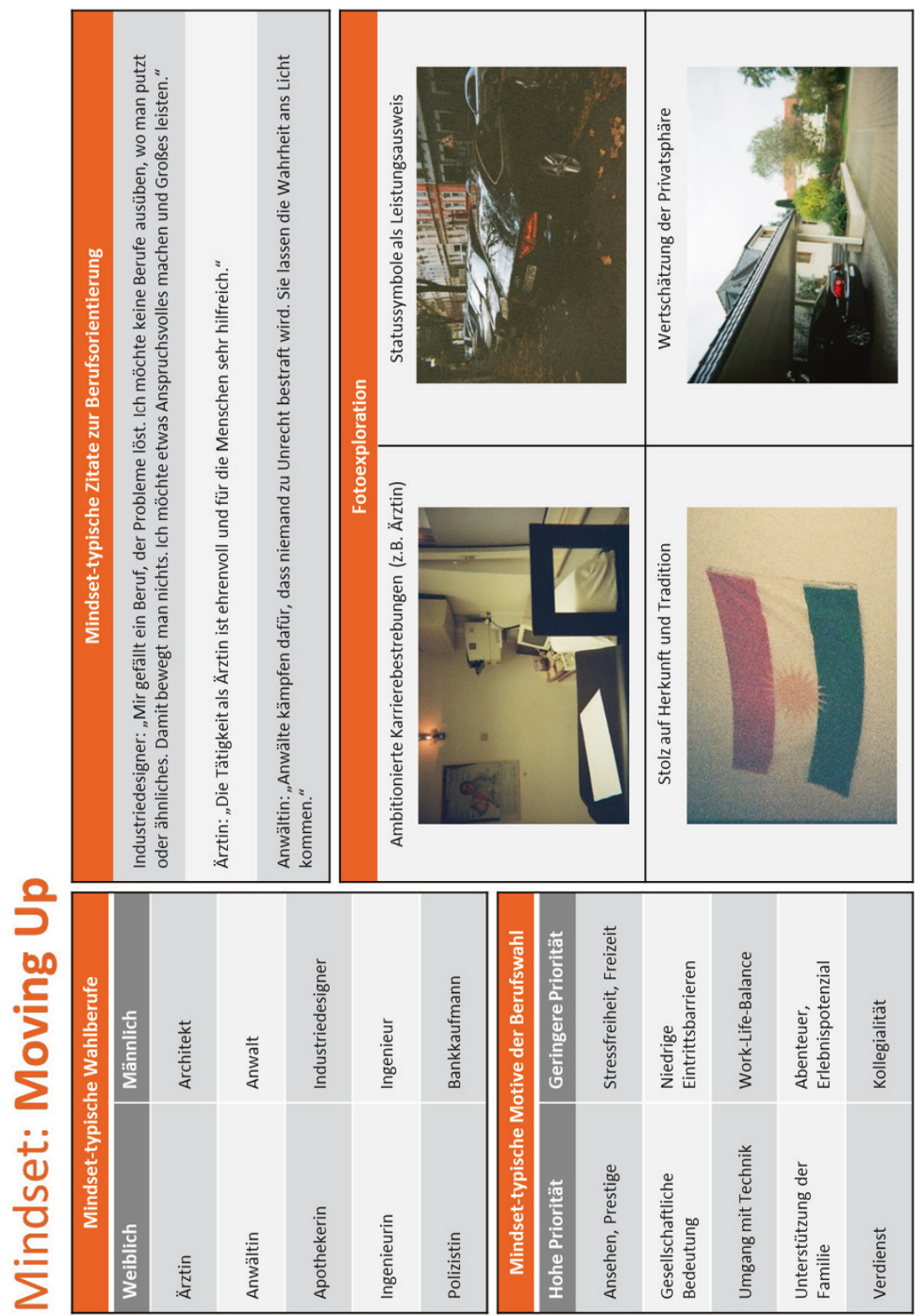


\subsubsection{Mindset-Profil: Breaking Free}

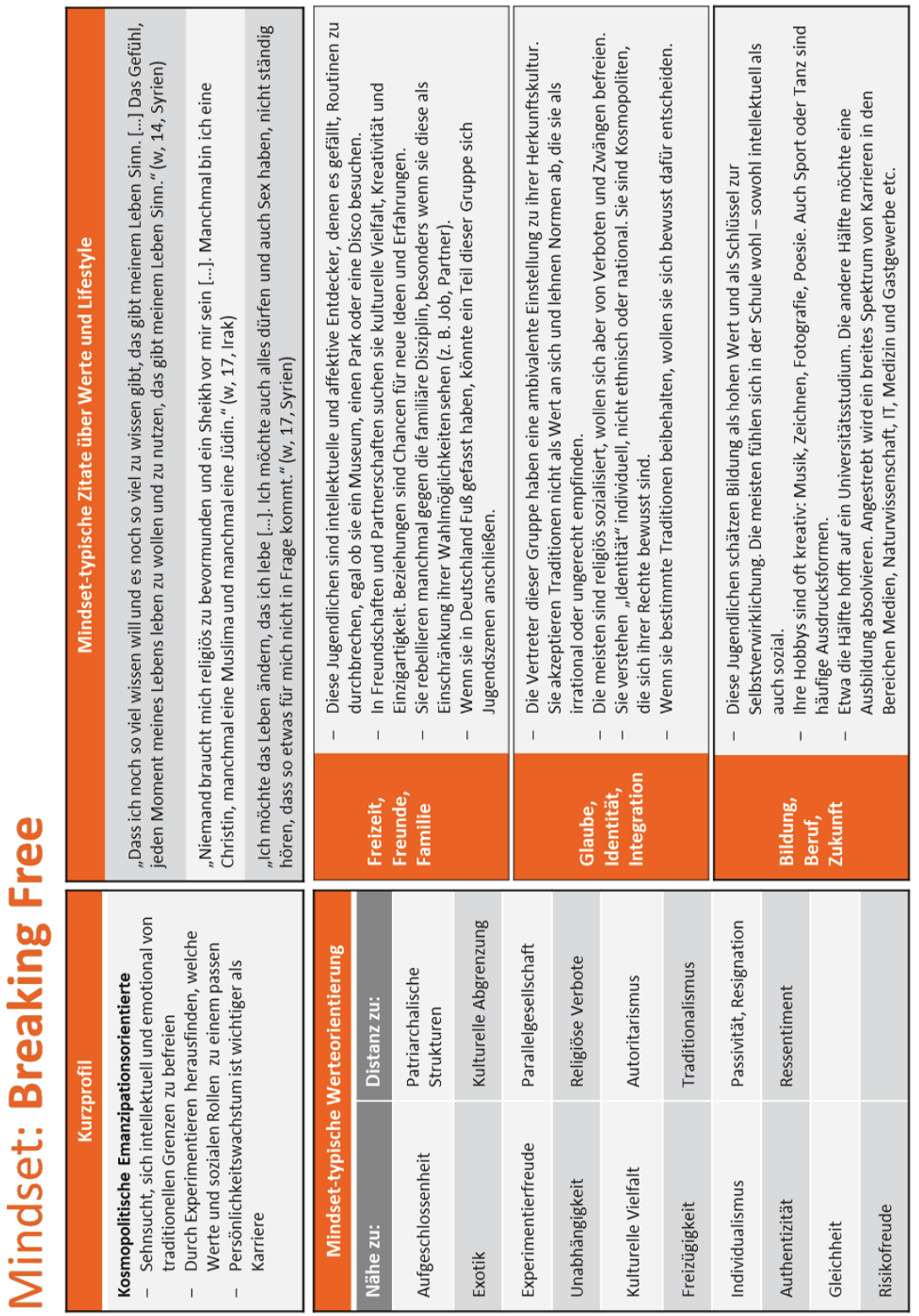




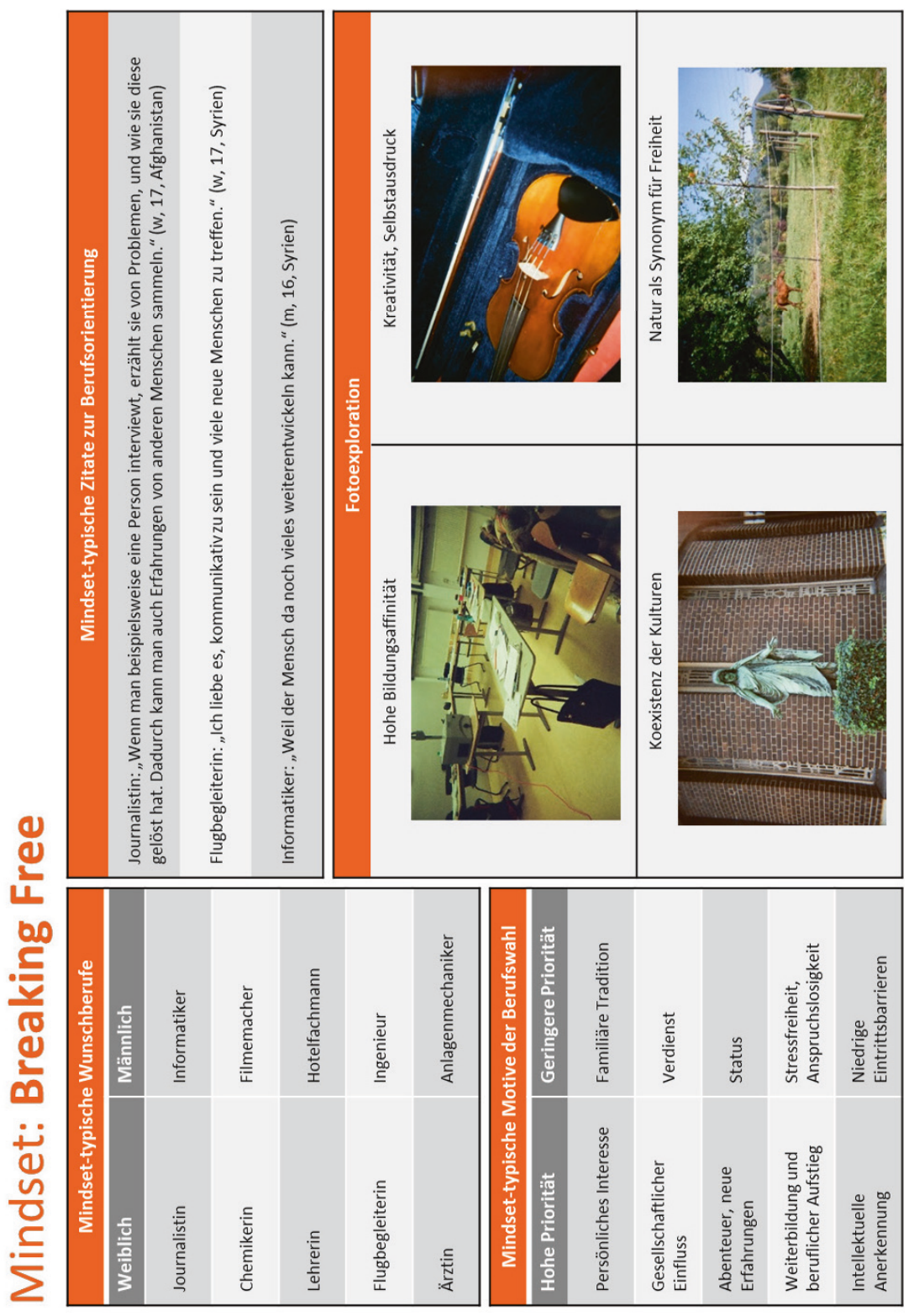




\subsubsection{Mindset-Profil: Holding On}

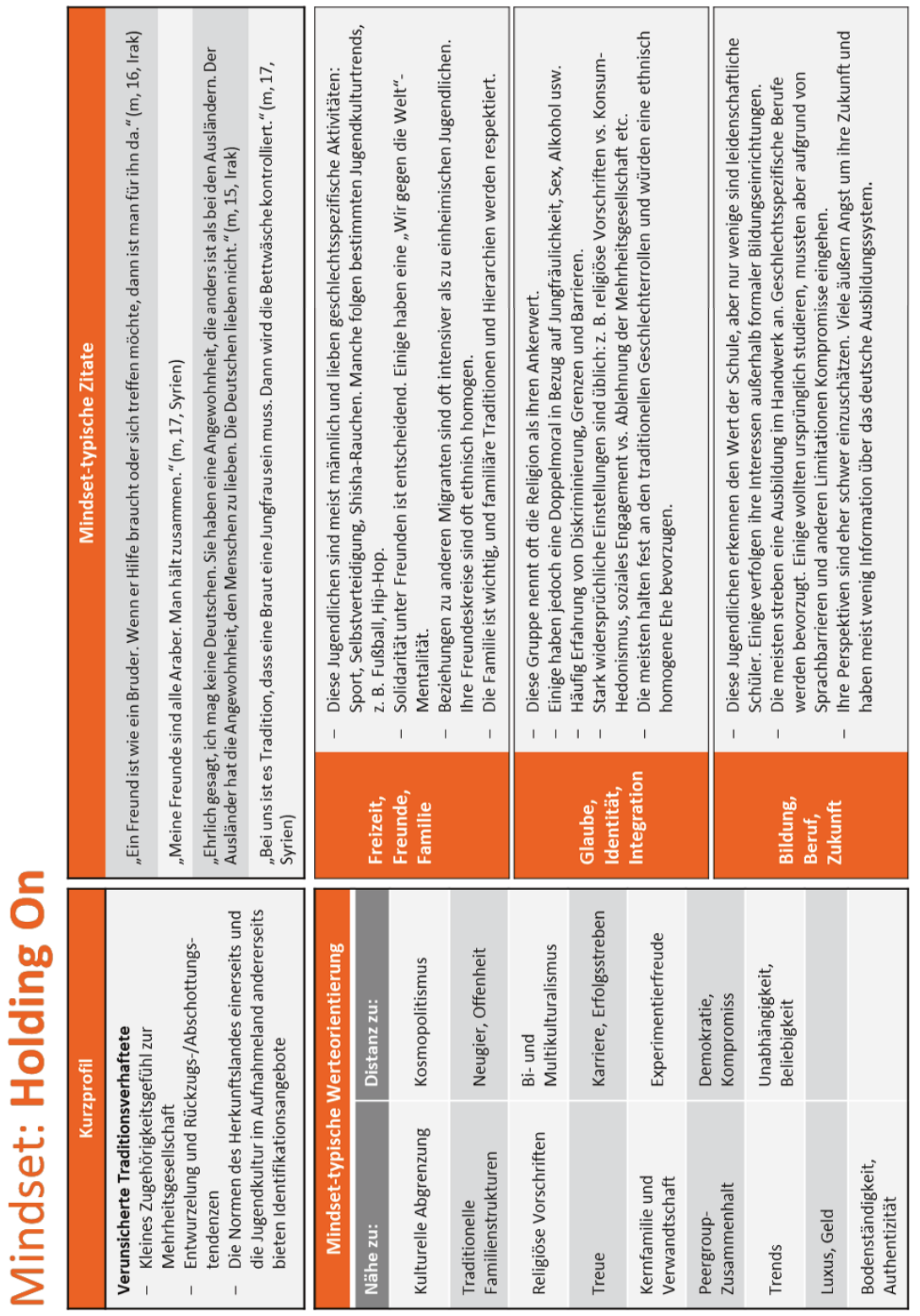



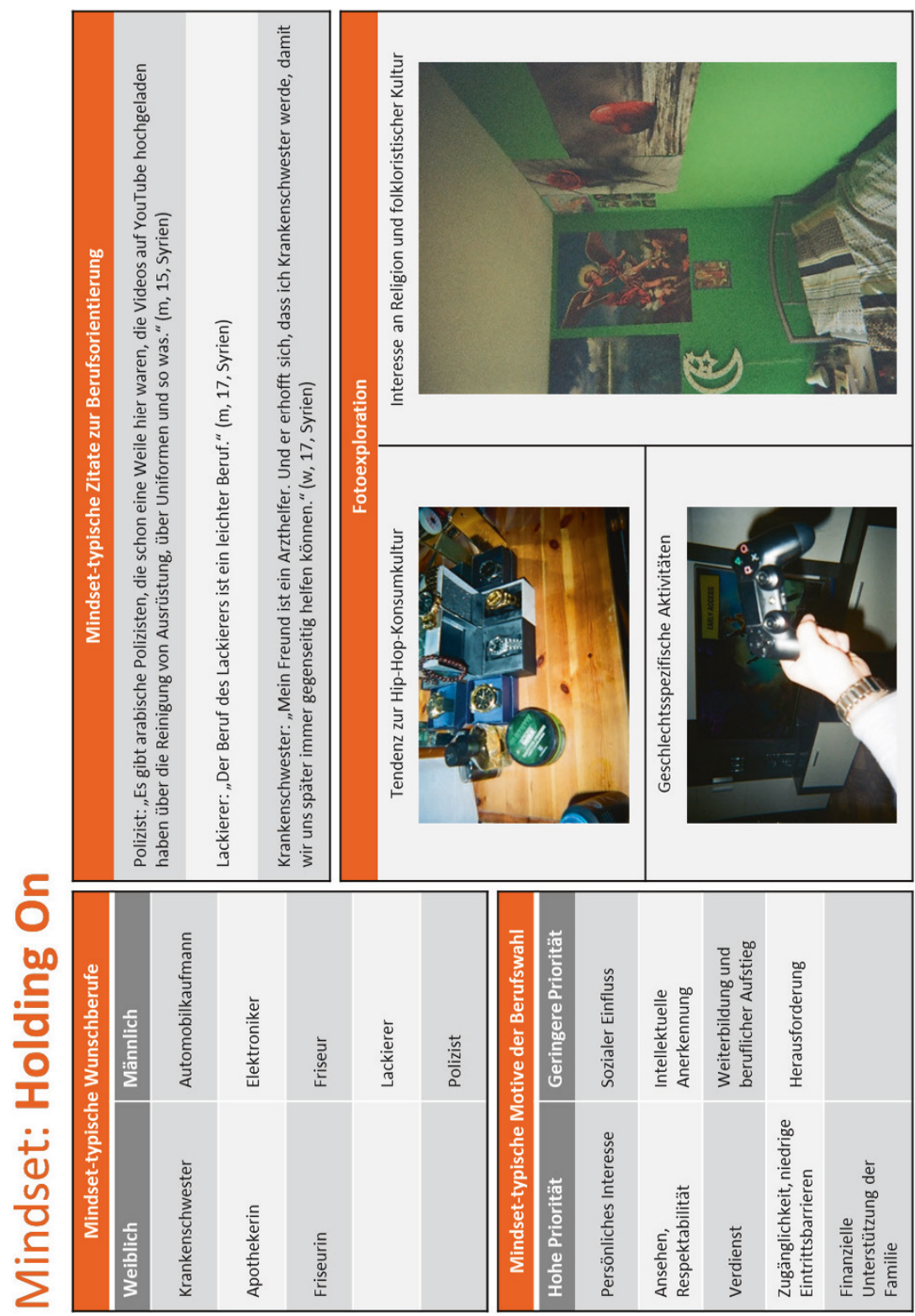


\subsubsection{Mindset-Profil: Blending In}
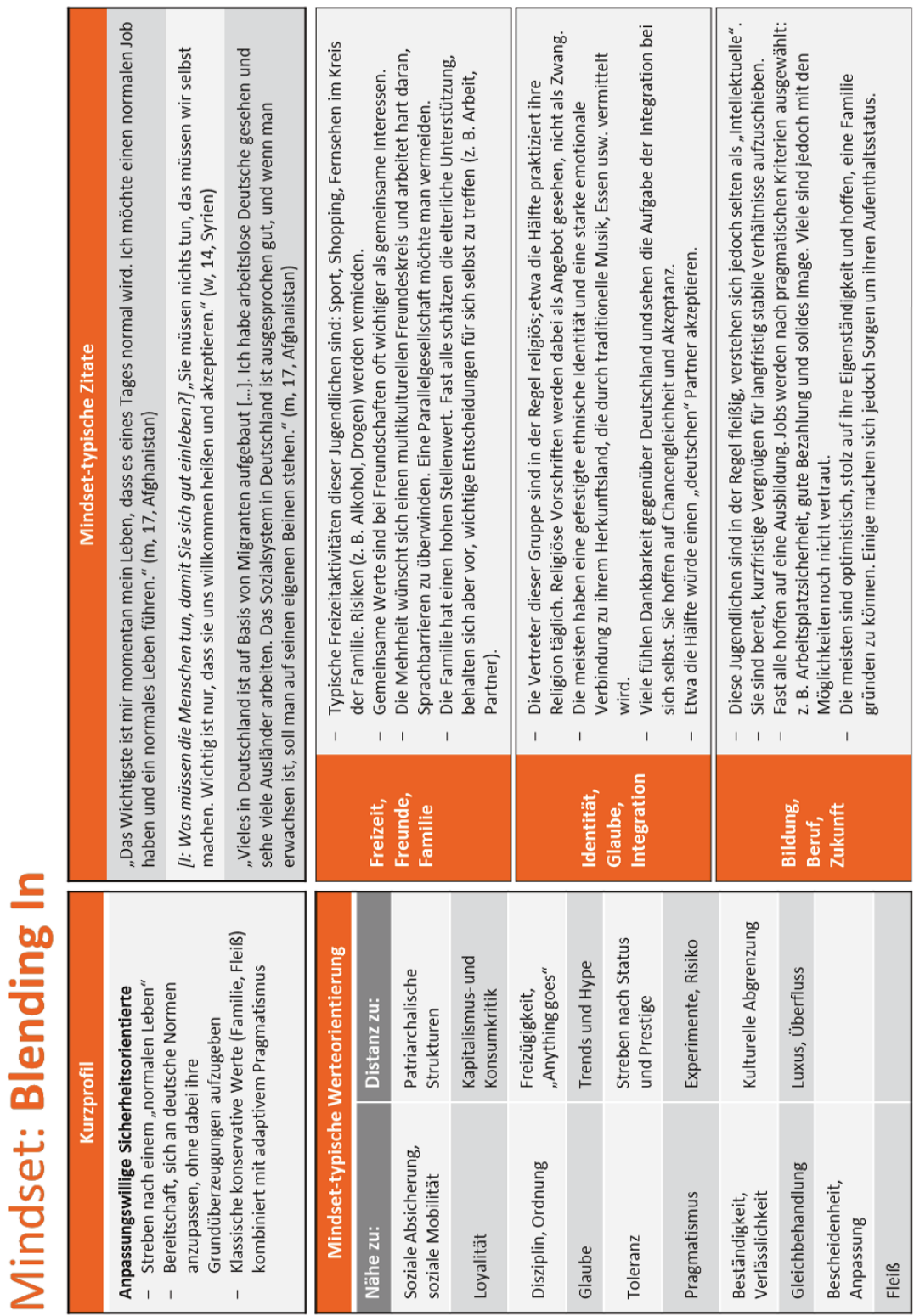

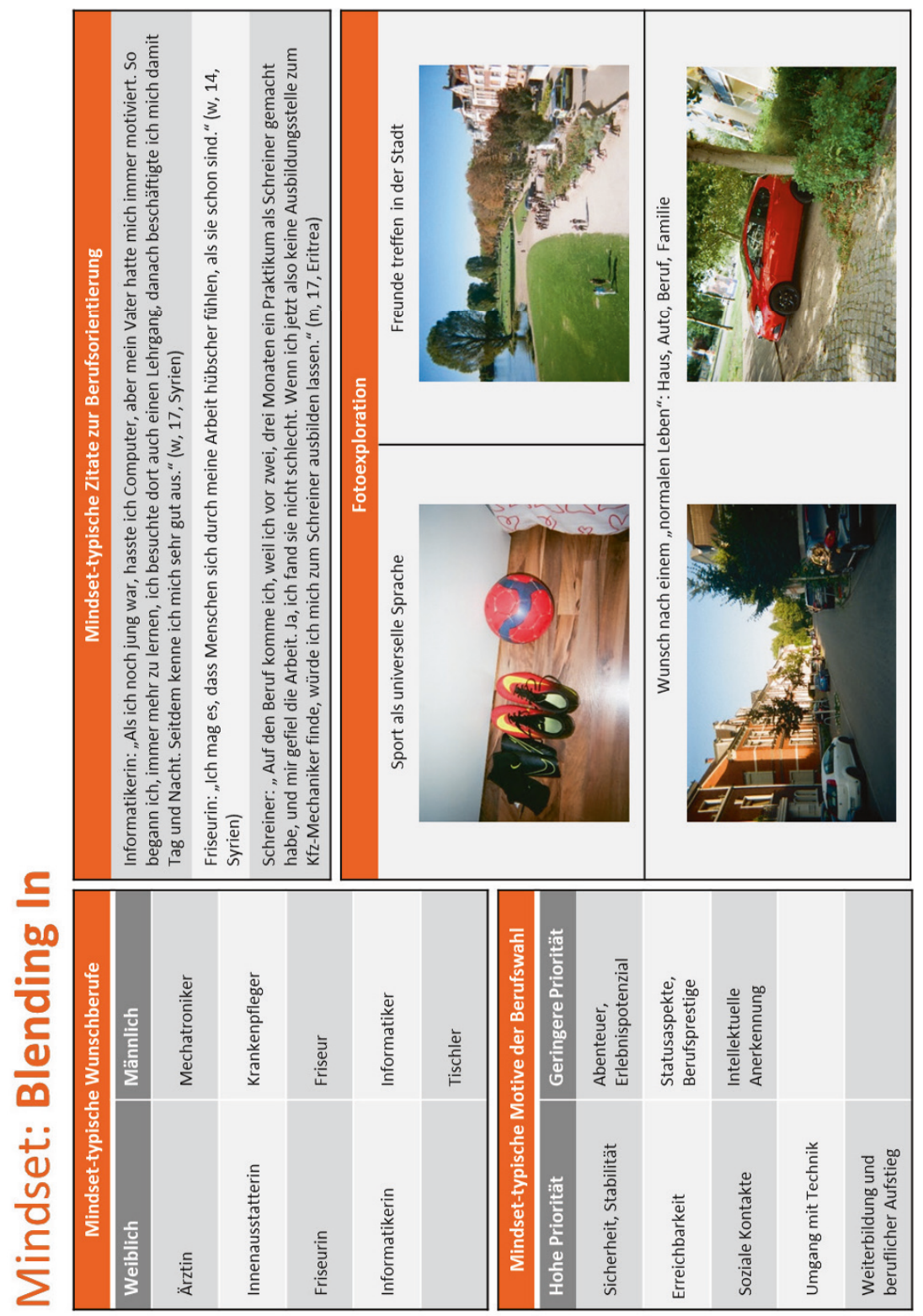
Open Access Dieses Kapitel wird unter der Creative Commons Namensnennung 4.0 International Lizenz (http://creativecommons.org/licenses/by/4.0/deed.de) veröffentlicht, welche die Nutzung, Vervielfältigung, Bearbeitung, Verbreitung und Wiedergabe in jeglichem Medium und Format erlaubt, sofern Sie den/die ursprünglichen Autor(en) und die Quelle ordnungsgemäß nennen, einen Link zur Creative Commons Lizenz beifügen und angeben, ob Änderungen vorgenommen wurden.

Die in diesem Kapitel enthaltenen Bilder und sonstiges Drittmaterial unterliegen ebenfalls der genannten Creative Commons Lizenz, sofern sich aus der Abbildungslegende nichts anderes ergibt. Sofern das betreffende Material nicht unter der genannten Creative Commons Lizenz steht und die betreffende Handlung nicht nach gesetzlichen Vorschriften erlaubt ist, ist für die oben aufgeführten Weiterverwendungen des Materials die Einwilligung des jeweiligen Rechteinhabers einzuholen.

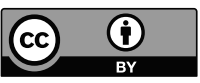

